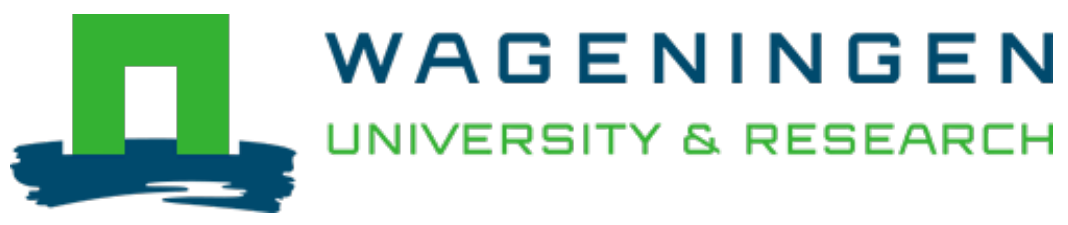

\title{
Gut microbiome stability and resilience : Elucidating the response to perturbations in order to modulate gut health
}

Gut

Fassarella, Marina; Blaak, Ellen E.; Penders, John; Nauta, Arjen; Smidt, Hauke et al

https://doi.org/10.1136/gutjnl-2020-321747

This article is made publicly available in the institutional repository of Wageningen University and Research, under the terms of article $25 \mathrm{fa}$ of the Dutch Copyright Act, also known as the Amendment Taverne. This has been done with explicit consent by the author.

Article 25 fa states that the author of a short scientific work funded either wholly or partially by Dutch public funds is entitled to make that work publicly available for no consideration following a reasonable period of time after the work was first published, provided that clear reference is made to the source of the first publication of the work.

This publication is distributed under The Association of Universities in the Netherlands (VSNU) 'Article $25 \mathrm{fa}$ implementation' project. In this project research outputs of researchers employed by Dutch Universities that comply with the legal requirements of Article $25 \mathrm{fa}$ of the Dutch Copyright Act are distributed online and free of cost or other barriers in institutional repositories. Research outputs are distributed six months after their first online publication in the original published version and with proper attribution to the source of the original publication.

You are permitted to download and use the publication for personal purposes. All rights remain with the author(s) and / or copyright owner(s) of this work. Any use of the publication or parts of it other than authorised under article $25 \mathrm{fa}$ of the Dutch Copyright act is prohibited. Wageningen University \& Research and the author(s) of this publication shall not be held responsible or liable for any damages resulting from your (re)use of this publication.

For questions regarding the public availability of this article please contact openscience.library@wur.nl 


\title{
Gut microbiome stability and resilience: elucidating the response to perturbations in order to modulate gut health
}

\author{
Marina Fassarella, ${ }^{1}$ Ellen E Blaak, ${ }^{2}$ John Penders, ${ }^{3}$ Arjen Nauta, ${ }^{4}$ Hauke Smidt, ${ }^{1}$ \\ Erwin G Zoetendal (1) ${ }^{1}$
}

'Laboratory of Microbiology, Wageningen University \& Research, Wageningen, The Netherlands

${ }^{2}$ Department of Human Biology, Maastricht University Medical Centre+, Maastricht, The Netherlands

${ }^{3}$ Department of Medical Microbiology, Maastricht University Medical Centre+, Maastricht, The Netherlands ${ }^{4}$ FrieslandCampina, Amersfoort, The Netherlands

Correspondence to Dr Erwin G Zoetendal, Laboratory of Microbiology, Wageningen University \& Research, Wageningen 6708 WE, The Netherlands; erwin.zoetendal@wur.n

Received 18 June 2020 Revised 9 September 2020 Accepted 17 September 2020 Published Online First 13 October 2020

\section{ABSTRACT}

The human gut microbiome is a complex ecosystem, densely colonised by thousands of microbial species. It varies among individuals and depends on host genotype and environmental factors, such as diet and antibiotics. In this review, we focus on stability and resilience as essential ecological characteristics of the gut microbiome and its relevance for human health. Microbial diversity, metabolic flexibility, functional redundancy, microbe-microbe and host-microbe interactions seem to be critical for maintaining resilience. The equilibrium of the gut ecosystem can be disrupted by perturbations, such as antibiotic therapy, causing significant decreases in functional richness and microbial diversity as well as impacting metabolic health. As a consequence, unbalanced states or even unhealthy stable states can develop, potentially leading to or supporting diseases. Accordingly, strategies have been developed to manipulate the gut microbiome in order to prevent or revert unhealthy states caused by perturbations, including faecal microbiota transplantation, supplementation with probiotics or non-digestible carbohydrates, and more extensive dietary modifications. Nevertheless, an increasing number of studies has evidenced interindividual variability in extent and direction of response to diet and perturbations, which has been attributed to the unique characteristics of each individual's microbiome. From a clinical, translational perspective, the ability to improve resilience of the gut microbial ecosystem prior to perturbations, or to restore its equilibrium afterwards, would offer significant benefits. To be effective, this therapeutic approach will likely need a personalised or subgroupbased understanding of individual genetics, diet, gut microbiome and other environmental factors that might be involved.

\section{INTRODUCTION}

The human gut microbiome is a diverse and complex ecosystem, inhabited by thousands of microbial species which coevolved within the host and are known to play an important role in health and disease. ${ }^{12}$ Gut microbiota composition varies among individuals and throughout development, and is dependent on host genotype and environmental factors. ${ }^{3}{ }^{4}$ Early microbial exposure, diet, age, geography and antibiotics exposure have all been implicated in the onset and maintenance of microbial diversity in the gut. ${ }^{5}$ Among those,

\section{Significance of this study}

What is already known on this subject?

- Perturbations to the human gut microbiome can disrupt the stability of the ecosystem, possibly resulting in recalcitrant (resilient) unhealthy states associated with diseases.

- The responses of the gut microbiome to perturbations and modulatory strategies are highly individual-specific.

What are the new findings?

- The tipping points that mark the critical switch to unhealthy states of the gut microbiome following perturbations are only beginning to be elucidated.

- Strategies to modulate the gut microbiome towards healthy resilient states are in their infancy.

How might it impact on clinical practice in the foreseeable future?

- Understanding the mechanisms shaping and determining gut microbiome resilience will allow the development of modulatory strategies to increase resilience of healthy states, or rather decrease and ultimately overcome resilience of unhealthy states.

- The future of microbiome-based medicine should comprise the characterisation of individual gut microbiomes and personalised strategies, with the expansion of nextgeneration 'omics' tools and sophisticated statistics methods to the clinical practice.

particular attention has been given to diet and its role in shaping gut microbiota composition. ${ }^{67}$

Although the gut microbiome is constantly exposed to external challenges, it has the ability to restore its equilibrium after a perturbation, such as infection with a pathogen or antibiotic therapy. ${ }^{8}$ This capacity for self-regeneration is also known as the resilience phenomenon ${ }^{9}$ (table 1). The acquisition of an unhealthy microbiota that has a high resilience potential may contribute to the chronicity of human microbiota-related diseases, ${ }^{10}$ including obesity and insulin resistance, ${ }^{11}$ type 2 diabetes mellitus (T2DM), ${ }^{12}$ gastrointestinal disorders ${ }^{13}$ and recurrent infection with Clostridioides difficile (formerly named Clostridium difficile). ${ }^{14}$ 
Table 1 Terms and definitions used in this review

\begin{tabular}{|c|c|c|}
\hline Terminologies & Definition & References \\
\hline $\begin{array}{l}\text { Alternative healthy state (of } \\
\text { the gut microbiome) }\end{array}$ & $\begin{array}{l}\text { A health-associated microbial composition and functionality, which develops after a perturbation and } \\
\text { differs from the initial state }\end{array}$ & Sommer et al $l^{9}$ \\
\hline $\begin{array}{l}\text { Faecal microbiota } \\
\text { transplantation }\end{array}$ & $\begin{array}{l}\text { Transfer of faeces suspension from a 'healthy' donor to a recipient who harbours an altered gut } \\
\text { microbiota associated with disease }\end{array}$ & van Nood et al $1^{4}$ \\
\hline Functional redundancy & $\begin{array}{l}\text { Measures the number of different populations within a community that are able to perform the same } \\
\text { functions }\end{array}$ & Blakeley-Ruiz et $a^{32}$ \\
\hline Gut microbiome & $\begin{array}{l}\text { The entire gut ecosystem, including all the microorganisms (bacteria, archaea, lower and higher } \\
\text { eukaryotes, as well as viruses), their genomes, associated functions and actual activity and the } \\
\text { surrounding environmental conditions }\end{array}$ & Marchesi and Ravel ${ }^{94}$ \\
\hline Gut microbiota & Collection of microorganisms present in the gut environment & Marchesi and Ravel ${ }^{94}$ \\
\hline $\begin{array}{l}\text { Healthy state (of the gut } \\
\text { microbiome) }\end{array}$ & $\begin{array}{l}\text { A health-associated state, characterised by a high microbial diversity, which favours functional diversity } \\
\text { and microbe-microbe and host-microbe interactions. Also referred as equilibrium state, balanced state } \\
\text { or homeostatic state }\end{array}$ & Byndloss et $a P^{54}$ and Lloyd-Pric et $a P^{56}$ \\
\hline Metabolic flexibility & $\begin{array}{l}\text { Capacity of a given species to metabolise distinct substrates, in adaptation to changes in the nutritional } \\
\text { environment }\end{array}$ & Rowland et al ${ }^{7}$ \\
\hline Microbial diversity & The number and abundance of distinct types of microorganisms in a particular ecosystem & Human Microbiome Project Consortium² \\
\hline Non-digestible carbohydrate & $\begin{array}{l}\text { A carbohydrate that resists host digestion, and thus reaches the colon where it can be metabolised by } \\
\text { microbes. Also referred to as dietary fibre. Certain NDCs are classified as prebiotics }\end{array}$ & Gibson et $a l^{95}$ \\
\hline Opportunistic pathogens & $\begin{array}{l}\text { Microbial symbionts or organisms from the environment that can become pathogenic following a } \\
\text { perturbation to the gut ecosystem }\end{array}$ & Buffie and Pamer ${ }^{37}$ \\
\hline Perturbation & $\begin{array}{l}\text { An external event that causes a distinct selective pressure on the ecosystem, also called a disturbance. } \\
\text { It can be either: } \\
\text { Pulse: relatively discrete, short-term perturbation } \\
\text { Press: long-term or continuous perturbation }\end{array}$ & Sommer et $a^{9}$ and Relman ${ }^{24}$ \\
\hline Prebiotic & A substrate that is selectively utilised by host microorganisms conferring a health benefit & Gibson et a $/^{95}$ \\
\hline Probiotic & $\begin{array}{l}\text { Live microorganisms, which when administered in adequate amounts confer a health benefit on the } \\
\text { host }\end{array}$ & Hill et a ${ }^{96}$ \\
\hline Resilience & $\begin{array}{l}\text { The property of a microbial ecosystem that defines how fast, and to what extent it will recover its initial } \\
\text { functional or taxonomical composition following perturbation }\end{array}$ & Sommer et $a l^{9}$ \\
\hline Resistance & Capacity of an ecosystem to remain unchanged on perturbation & Sommer et $a l^{9}$ \\
\hline Stability & $\begin{array}{l}\text { Attribute of a given ecosystem to maintain a state of equilibrium and resist perturbations, or to show } \\
\text { resilience after a perturbation }\end{array}$ & Lozupone et a l $^{23}$ \\
\hline Tipping points & $\begin{array}{l}\text { Critical aspects that determine the switch of the gut microbiota composition and/or functionality to a } \\
\text { different state following a perturbation }\end{array}$ & Lahti et $\left.a\right|^{27}$ \\
\hline $\begin{array}{l}\text { Unhealthy state (of the gut } \\
\text { microbiome) }\end{array}$ & $\begin{array}{l}\text { Ecosystem often characterised by a low microbial diversity, with a depletion of health-associated } \\
\text { microbes and expansion of pathogens; state associated with disease, also called dysbiosis }\end{array}$ & Brüssow $^{60}$ \\
\hline Xenobiotics & $\begin{array}{l}\text { Foreign molecules to the human body, comprising among others food additives, pharmaceuticals and } \\
\text { environmental chemicals }\end{array}$ & Atashgahi et $a P^{53}$ \\
\hline
\end{tabular}

NDCs, non-digestible carbohydrates.

Understanding mechanisms that confer resilience to stable states of the gut microbiota is urgently needed to allow the development of modulatory strategies to increase resilience of healthy states, or rather decrease or overcome resilience of unhealthy states. ${ }^{15}$ In order to achieve this, approaches comprising a personalised understanding of gut microbiome, individual genetics, diet and other environmental factors would be necessary. ${ }^{1617}$ Therefore, the aim of this review is to give an overview of potential mechanisms involved in gut microbiome resilience that may govern the response to perturbations, and to determine whether dietary components can shape gut microbiota composition and function towards resilient healthy states.

\section{Human gut microbiome: an overview}

The microbial cells that colonise the human body are at least as abundant as the somatic cells and collectively contribute far more non-redundant genes than the human genome. ${ }^{1}$ Zooming in to the gut ecosystem of a single individual, it is estimated that over 1000 bacterial species, with a collective metagenome of several million genes, reside in the gut. ${ }^{18}$ The predominant bacterial phyla are Actinobacteria, Bacteroidetes, Firmicutes,
Proteobacteria and Tenericutes, while Fusobacteria, Saccharibacteria, Spirochaetes, Synergistetes and Verrucomicrobia are present at lower abundance. ${ }^{19}$

Various studies have indicated the existence of a core gut microbiota, comprising prevalent gut microbial populations, genes and essential functions for bacterial life in the ecosystem, which are observed in most individuals and persist over time. ${ }^{20}$ Key microbial species have shown to be conserved in the gut, despite regular fluctuations in their abundances, potentially contributing to the maintenance of a health-promoting, mutualistic microbiota configuration. ${ }^{21} 22$

\section{Stability, resistance and resilience of the human gut microbiome}

Stability, resistance and resilience are essential ecological characteristics of the gut microbiome, given its importance for human health. ${ }^{23}$ In the absence of a perturbation, the gut microbial community oscillates around a stable ecological state, showing a dynamic equilibrium. ${ }^{24}$ The gut microbiota is often highly resilient to perturbations, allowing a host to keep key species for 


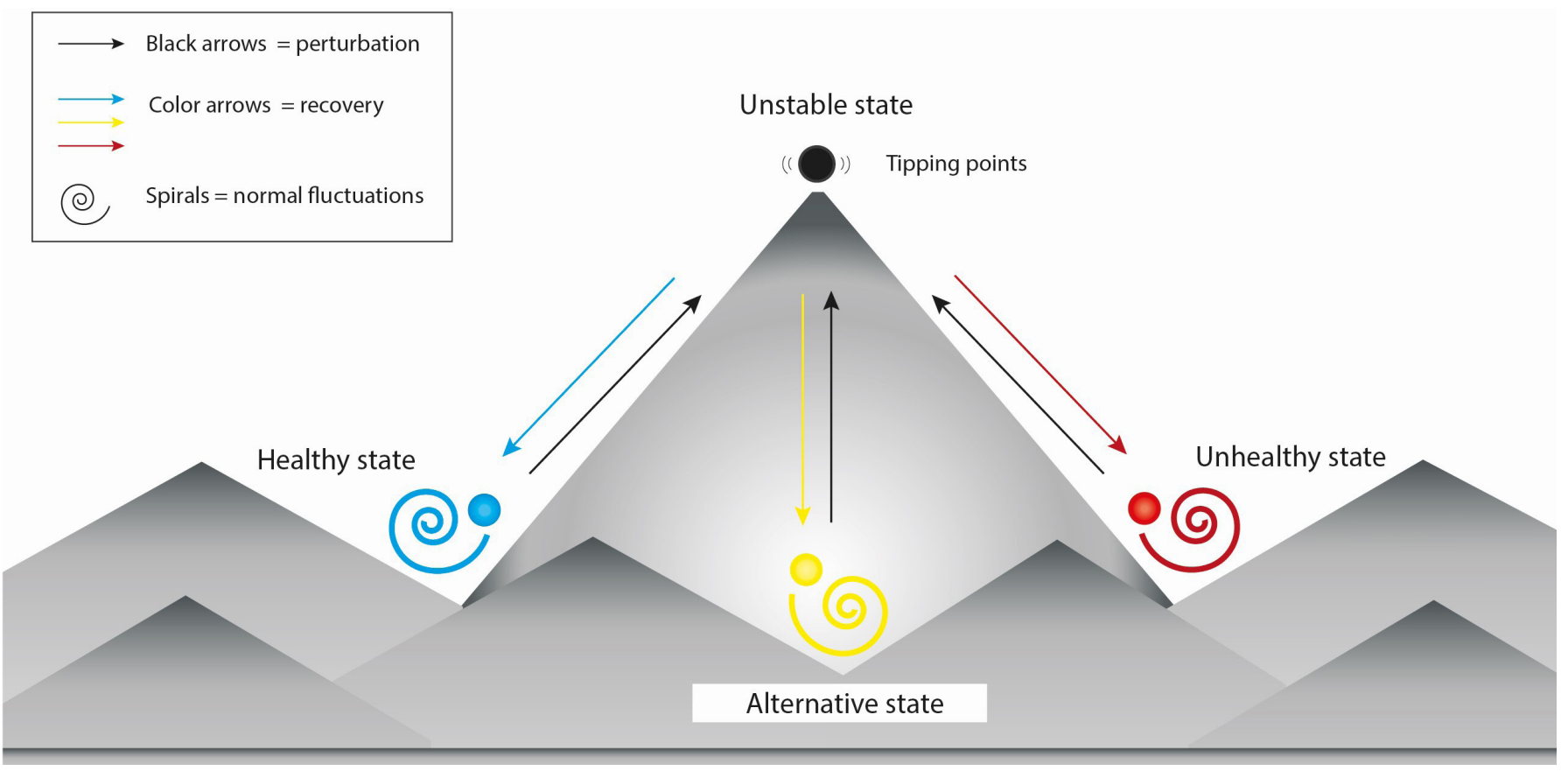

Figure 1 Gut microbiome resilience landscape. The adult gut microbiome comprises a stable, yet also dynamic system, which can be represented in a landscape. The valleys correspond to stable states, around which the gut microbiota oscillates. Three stable states of the gut microbiome are depicted in this figure: healthy, alternative (healthy) and unhealthy. On perturbations, the stable ecosystem can be pushed towards an unstable and transient state, represented by the peak, after which it will recover to either its initial state or to another stable state. The extent and speed of recovery will depend on tipping points, which are critical aspects that determine the switch between alternative states of gut microbiota composition and/or functionality.

long periods. In healthy adults, the gut microbiota composition can be stable for years, ${ }^{25}$ although the relative abundance of each microbe fluctuates over time, and it varies between individuals and within individuals over the course of their lives. ${ }^{6}$ Consequently, it is an open empirical question as to how many and which species actually provide benefits and whether or not they are associated with gut microbiome stability and resilience.

Although some reviews have extensively discussed resilience concepts, ${ }^{23-25}$ and predictive models have been used for a better understanding of resilience in silico, ${ }^{26}$ applying them to the complex gut ecosystem is still challenging. The threshold for transitions of the gut microbiota to different states is not yet clear, and which tipping points ${ }^{27}$ mark the critical switch to stable unhealthy states following perturbations are only beginning to be elucidated. To this end, the knowledge gained with respect to resilience in other ecosystems can be used as a powerful tool in this progress. ${ }^{28} 29$

Elucidating the response of the gut microbiome to perturbations can shed light on the mechanisms of resilience presented by an individual microbial ecosystem. In this context, the duration and severity of perturbations, which are classified either as pulses or presses, will greatly determine its impact on the microbial community. ${ }^{24}$ External perturbations can lead the stable microbial ecosystem to be pushed towards an unstable and transient state, after which it might recover to its initial state. However, they can also lead to the formation of an alternative stable state (healthy), or to an unhealthy stable state associated with diseases. Figure 1 represents these transitions in the form of a landscape.

A critical aspect for conferring stability and resilience to the gut microbiome is microbial diversity, which seem to be governed by several selective pressures within host habitats. ${ }^{30}$ A high microbial diversity results in an increased level of functional redundancy, which is generally believed to play a role in stabilising microbiota functions during perturbations, and thus supporting resilience. ${ }^{31}$ The coexistence of microorganisms with a similar role in the ecosystem may be positive for the host, since other microbes can compensate for the loss of a beneficial strain by providing the same benefit. In fact, two given microbial communities that differ in compositional terms, may behave very similarly in functional terms, yielding comparable protein and metabolite profiles. ${ }^{32}$

Which microbial species persist in the gut will depend on host control, together with their ability to compete within the set of ecological networks existing in the microbial ecosystem. ${ }^{33}$ Microbial interactions, ranging from mutualism and commensalism all the way to competition and amensalism, as well as the symbiotic relationship between microbes and their host can be considered essential factors in shaping resilience. ${ }^{34}$ To date, most gut microbial interactions described in the literature involve bacteria, and to some extent, archaea. However, increasing attention has been given to the role of viruses ${ }^{35}$ and eukaryotes, particularly parasites, ${ }^{36}$ in shaping gut microbiota composition via interactions with other microorganisms. An overview of various types of potential interactions in the gut is depicted in figure 2 .

A stable microbial community can resist the invasion of nonnative bacteria and the expansion of opportunistic pathogens, resulting in the phenomenon known as colonisation resistance. ${ }^{37}$ The capacity of forming biofilms within the gut can be a fundamental aspect in this process, offering several competitive advantages, such as increased bacterial residence time by providing protection from stressors, and enhanced bacteria-host synergy, with improved exchange of nutrients between them. ${ }^{38}$ While the formation of biofilms by beneficial bacteria in the gut is still being investigated, biofilms in the unhealthy gut are well documented, such as in the case of IBD, in which they have been shown to be formed by Bacteroides fragilis. ${ }^{39}$ 


\begin{tabular}{|c|c|c|c|}
\hline $\begin{array}{l}\text { Microbial } \\
\text { interactions }\end{array}$ & Description & $\begin{array}{l}\text { Effects on gut microbiome } \\
\text { stability and resilience }\end{array}$ & $\begin{array}{c}\text { Examples in the gut } \\
\text { ecosystem }\end{array}$ \\
\hline Amensalism & $\begin{array}{l}\mathrm{A} \rightleftharpoons-\mathrm{B} \\
\text { Production of molecules } \\
\text { that harm other microbes }\end{array}$ & $\begin{array}{l}\text { - Promotes colonisation resistance } \\
\text { and prevents invasion of } \\
\text { pathogens }\end{array}$ & $\begin{array}{l}\text { - Production of } \\
\text { antimicrobials [45] }\end{array}$ \\
\hline $\begin{array}{l}\text { Antagonistic } \\
\text { coevolution }\end{array}$ & $\begin{array}{c}\bigcap_{\Delta} \stackrel{\text { DNA }}{\longrightarrow} \underbrace{\longrightarrow}_{\text {Bacteria }} \bigcirc \\
\begin{array}{c}\text { Reciprocal evolution of } \\
\text { host defence and parasite } \\
\text { infectivity }\end{array}\end{array}$ & $\begin{array}{l}\text { - Transfer of genes that increase } \\
\text { fitness of the bacteria in the gut } \\
\text { - Maximises or minimises the } \\
\text { efficiency of nutrient metabolism } \\
\text { by bacteria } \\
\text { - Promotes microbial diversity }\end{array}$ & $\begin{array}{l}\text { - Coevolution, and } \\
\text { horizontal gene transfer } \\
\text { by lysogenic } \\
\text { (temperate) } \\
\text { bacteriophages [35] }\end{array}$ \\
\hline Competition & $\begin{array}{l}\mathrm{A} \rightleftharpoons \mathrm{B} \\
\begin{array}{l}\text { One outcompetes the other } \\
\text { for resources or space }\end{array}\end{array}$ & $\begin{array}{l}\text { - Selection for species/ strains } \\
\text { that are better suited to colonise } \\
\text { the gut } \\
\text { - Persistence in the gut, when } \\
\text { able to outcompete and colonise } \\
\text { surfaces }\end{array}$ & $\begin{array}{l}\text { - Iron uptake [85] } \\
\text { - Adhesion to epithelial } \\
\text { cells [38] }\end{array}$ \\
\hline Commensalism & $\begin{array}{c}\mathrm{A} \longrightarrow+\mathrm{B} \\
\begin{array}{c}\text { One benefits, while the } \\
\text { other is apparently } \\
\text { unaffected }\end{array}\end{array}$ & $\begin{array}{l}\text { - Ideal environment for microbial } \\
\text { growth, with provision of nutrients } \\
\text { and protection, allowing microbe- } \\
\text { microbe interactions } \\
\text { - Modulation of host immune } \\
\text { tolerance }\end{array}$ & $\begin{array}{l}\text { - Colonisation of } \\
\text { mucosal surfaces [37] } \\
\text { - Utilisation of } \\
\text { microbially produced } \\
\text { acetate to produce } \\
\text { butyrate [81] }\end{array}$ \\
\hline Cooperation & $\begin{array}{c}\mathrm{A}+\rightleftharpoons+\mathrm{B} \\
\Longleftrightarrow \begin{array}{c}\text { Not-obligatory co- } \\
\text { dependence among } \\
\text { symbionts }\end{array}\end{array}$ & $\begin{array}{l}\text { - Collective response to } \\
\text { environmental perturbations } \\
\text { - Adaptation to changes, by using } \\
\text { the complementary nutrient- } \\
\text { degrading capabilities of each } \\
\text { other }\end{array}$ & $\begin{array}{l}\text { - Quorum-sensing [49] } \\
\text { - Cross-feeding } \\
\text { [42] }\end{array}$ \\
\hline Mutualism & 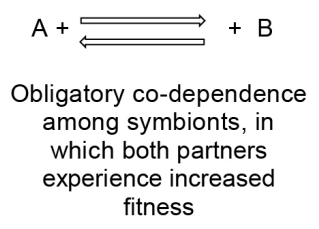 & $\begin{array}{l}\text { - The mutual benefits result in the } \\
\text { persistence of the microbes in the } \\
\text { gut, promoting stability }\end{array}$ & $\begin{array}{l}\text { - Digestion of complex } \\
\text { carbohydrates [7] } \\
\text { - Production of vitamins } \\
\text { [84] }\end{array}$ \\
\hline Parasitism & $\begin{array}{l}0 \Longrightarrow \text { Host } \\
\text { Parasite } \\
\begin{array}{l}\text { One organism benefits at } \\
\text { the expense of the other }\end{array}\end{array}$ & $\begin{array}{l}\text { - Selective pressure, inducing } \\
\text { changes in microbial composition }\end{array}$ & $\begin{array}{l}\text { - Protozoan infections, } \\
\text { production of } \\
\text { metabolites and } \\
\text { interactions with the } \\
\text { host immune system } \\
\text { [36] }\end{array}$ \\
\hline Predation & $\begin{array}{l}\text { Bacteria } \\
\text { Phages } \\
\text { One organism (predator) } \\
\text { engulfs or attacks the } \\
\text { other (prey), usually } \\
\text { resulting in the death of } \\
\text { the prey }\end{array}$ & $\begin{array}{l}\text { - Promotes microbial diversity, by } \\
\text { applying selective pressure } \\
\text { - Lysis of bacterial cells releases } \\
\text { nutrients into the environment }\end{array}$ & $\begin{array}{l}\text { - Lytic (virulent) } \\
\text { bacteriophages [35] }\end{array}$ \\
\hline
\end{tabular}

Figure 2 Microbial interactions and potential implications for gut microbiome stability and resilience. Microbial interactions existing in the gut are described, including the possible effects on the gut microbiome as well as examples of each type of interaction.

In line with that, the mucus layer covering the gut epithelium can act as a biofilm matrix, enabling adhesion and protecting the inhabiting microbes, which then become persistent and resilient in the gut environment. ${ }^{40}$ Furthermore, the mucus layer is a key contributor to the structural and functional stability of the gut microbiome and its tolerance by the host, playing a crucial role in the gut by spatially compartmentalising microorganisms to the lumen, besides producing host-derived antimicrobials and secretory IgA, which contributes to the exclusion of microorganisms from the epithelial surface. ${ }^{41}$ Besides that, mucin, a component of the mucus layer, is degraded as preferable carbon source by certain species, such as Akkermansia muciniphila, a prominent bacterium in the human gut. ${ }^{42}$ Other members of the gut microbiota, like Bacteroides thetaiotaomicron, are able to switch their metabolism to utilise host-derived glycans when dietary polysaccharides are scarce, showing a high metabolic flexibility, which also contributes to their persistence in the gut. ${ }^{43}$

Antagonism is another interaction among gut microbes that can prevent the overgrowth of specific species and thereby reduce the risk of opportunistic pathogens to flourish. This can 
be achieved by competition for nutrients, as well as amensalistic activity through the production of bacteriocins and toxic metabolites. ${ }^{44}$ Microcins produced by Escherichia coli in the gut, for example, exhibit activity against other members of Enterobacteriaceae. ${ }^{45}$ Microbial metabolites, like short-chain fatty acids (SCFAs), can also inhibit other microorganisms ${ }^{46}$ by either lowering the $\mathrm{pH}$ in the lumen, or through additional bactericidal and bacteriostatic mechanisms of action, protecting the gut against colonisation by sensitive pathogens. ${ }^{47}$

In contrast, many microbes employ cooperative traits to remain competitive within communities. Bacteria can use quorum sensing signalling molecules, called autoinducers, which function as a communication system to report on cell density, diffusion conditions and species composition of the surrounding environment, enabling the microbes to collectively modify behaviour in response to changes. ${ }^{48}$ Such communication within and between different microbial species can influence the network of interactions occurring in the ecosystem, and thus altering microbiota composition. ${ }^{49}$

Another particular type of interaction among gut microbes is known as cross-feeding or syntrophy, whereby microorganisms form highly efficient cooperative metabolic processes, in which they exchange nutrients or other compounds. ${ }^{50}$ Gut microorganisms can exploit the complementary nutrient-degrading and vitamin-production capabilities of each other, which sustain the production of metabolites for reciprocal exchanges. Mucus degradation and fermentation by A. muciniphila, for example, result in the liberation of oligosaccharides and subsequent production of acetate, which becomes available to other gut microbes. ${ }^{42}$ Bifidobacterial populations can also cooperate among themselves, as well as with other members of the gut microbiota by means of cross-feeding, in which they collectively use their extensive saccharolytic features to metabolise carbohydrates. ${ }^{51}$ Interspecies hydrogen $\left(\mathrm{H}_{2}\right)$ transfer is another example of a mutually beneficial process in the gut, in which one microorganism degrades organic compounds, such as polysaccharides, and releases reducing equivalents in the form of $\mathrm{H}_{2}$, which, in turn, are used by the second microorganism as electron donor. ${ }^{52}$

Besides interactions between microbes, host-microbe interactions are equally important for a stable and resilient microbiome, and are principally based on the same types of interactions as described above. The relationship between host, gut microbes and health are profound and intricate. The list of benefits provided by the microbiota to human health is increasing and includes improved food digestion and nutrition, ${ }^{7}$ regulation of human metabolism and immune system, ${ }^{37}$ prevention of colonisation and invasion of the host by pathogens,${ }^{44}$ as well as xenobiotic metabolism. ${ }^{53}$ At the same time, the indigenous microbes benefit from this symbiosis, which involves the acquisition of nutrients, habitat and an effective means of dispersal. ${ }^{24}$

Host-microbiota interactions are site-specific and determine intestinal function and physiology. Recent insights suggest that hosts are under strong natural selection to shape their microbiota to be beneficial. ${ }^{33}$ For this reason, the human host might be able to control microbial numbers in the small intestine to avoid competition for simple sugars and amino acids, as well as limit oxygen availability in the large intestine to obtain microbial fermentation products from dietary fibres. ${ }^{54}$ Additionally, the acidic $\mathrm{pH}$ found in the stomach is considered a first line of protection against foreign microbes, ${ }^{55}$ and the bile acids, secreted at the proximal end of the small intestine, are bactericidal to certain species and known to broadly shape the composition of the microbiota. ${ }^{7}$ Furthermore, peristalsis is one of the greatest negative selection pressures in the gut, which removes millions of viable microbial cells, indicating that adhesion to human gut epithelium might be essential for the persistence in the environment. ${ }^{40}$

Interactions between the human host and the gut microbiota require a delicate balance that must be actively maintained by both host and microbe to achieve a healthy steady state. ${ }^{33}$ Potential factors involved in this balance are represented in figure 3 . In the context of stability and resilience, a healthy functional microbiome would comprise not a single static state but rather a dynamic ecosystem with the ability to recover to an equilibrium state after stress and perturbation. ${ }^{56}$ When the stability of healthy states is disrupted, unhealthy stable states can develop ultimately, with negative implications to the host. ${ }^{23}$

\section{Unhealthy states of the gut microbiome}

Among the factors implicated in the development of unhealthy states of the gut microbiome are drastic alterations in dietary patterns, microbial infections and high exposure to xenobiotics, including the extensive use of medications, particularly antibiotics. 5357

Unhealthy states of the gut microbiota are often characterised by significant compositional and/or functional microbiome alterations, with marked decrease in diversity, ${ }^{58}$ potentially resulting in a depletion of health-associated bacteria,${ }^{47}$ as well as an expansion of pathogenic microbes. ${ }^{45}{ }^{59}$ Unhealthy states of the gut microbiome are usually the consequence of a major perturbation and can either: (1) be transient and last for a short time, recovering to the initial healthy state prior to the perturbation; (2) develop into a stable unhealthy state with negative implications for the host, which consists of an altered composition and functionality that persists over time, making it resilient or resistant to perturbations in the form of (medical) treatments.

Resilient or resistant unhealthy states of the gut microbiome may explain the unsuccessful therapeutic interventions applied to microbiota-mediated conditions, and therefore have been associated with the chronicity of several diseases. Classic examples of such states are $\mathrm{IBD}^{13}$ and recurrent $C$. difficile infection. ${ }^{14}$ In these cases, the therapy itself can be considered a perturbation (a term not frequently used in the literature in this context), varying from supplementation of probiotics and prebiotics, antibiotics administration, as well as faecal microbiota transplantation, which usually are not sufficient to push the ecosystem towards a healthier stable state, meaning that it returns to the initial unhealthy state. ${ }^{9}$ A limiting aspect in defining these different states is the lack of sufficient evidence to distinguish the unhealthy state as a cause or consequence of disease. ${ }^{60}$

Stability and resilience in unhealthy states of the gut microbiome have not yet been fully understood, and more studies are necessary to determine why those ecosystems are resilient, despite being often less diverse. One possible explanation would be the presence/maintenance of the essential microbial functions in the gut, with an overexpression of functions associated with disease due to the high abundance of pathogens, as well as the establishment of a new set of microbial interactions, that ultimately make the ecosystem stable and therefore resilient.

\section{Antibiotics perturbation: impacts on the gut ecosystem}

Antibiotic therapy induces one of the most drastic perturbations to the gut microbiome, affecting not only the pathogens to which they are directed but also members of the microbiota. ${ }^{61}$ These impacts will depend greatly on the chemical nature of the drug, including the target spectrum, pharmacokinetic and pharmacodynamic properties, but also on dose and duration, route 


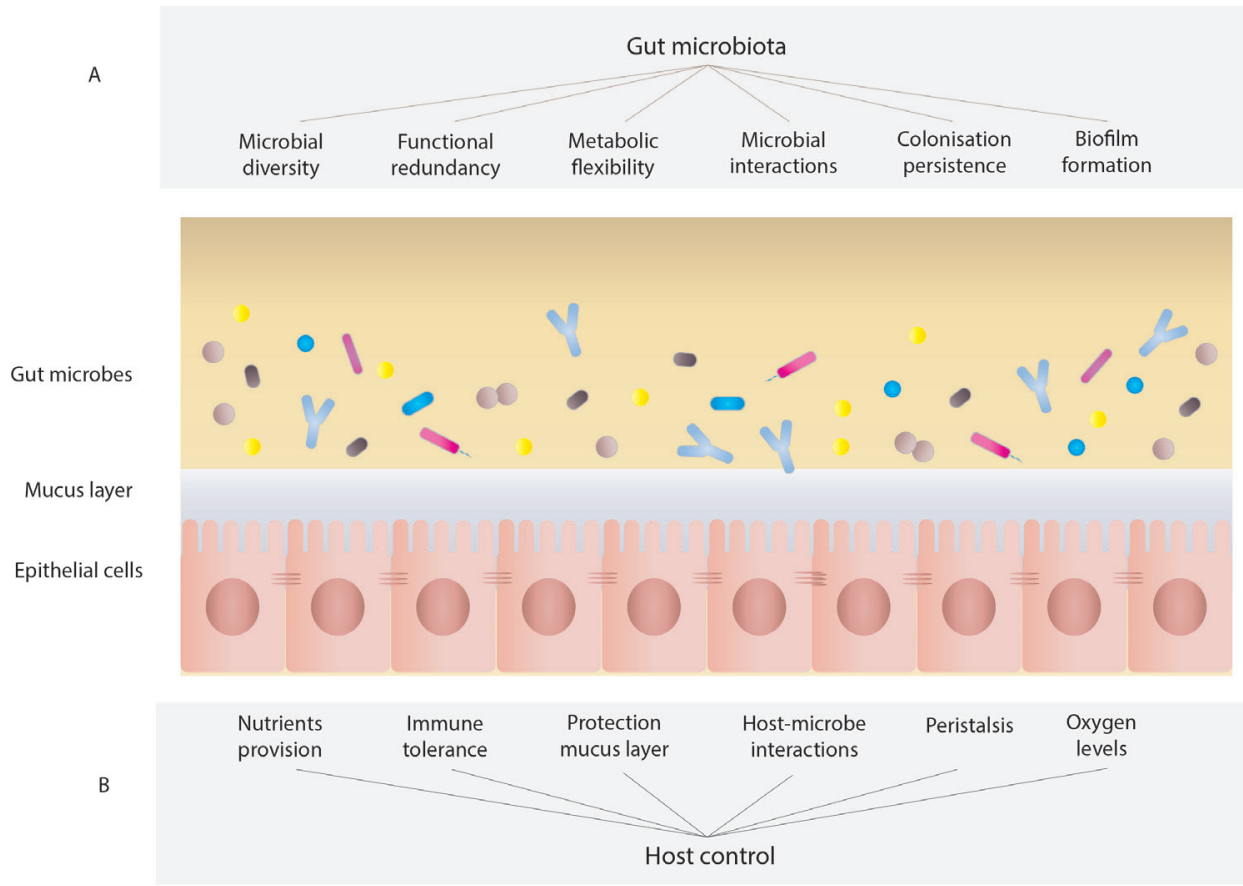

Figure 3 Characteristics of the host-microbiome holo-organism determining gut microbiome stability and resilience. Resilience of the gut microbiome seems to be governed by several intrinsic features, which shape microbial composition and consequently the characteristics of the gut ecosystem. These features can be classified into two groups-(A) characteristics of the gut microbiome: microbial diversity, functional redundancy, metabolic flexibility, microbial interactions, colonisation persistence, biofilm formation, all play a role in the maintenance of a stable ecosystem in the gut; (B) host control: various selective pressures exerted by the host, like the nutrients provided, immune tolerance, protection by the mucus layer, host-microbe interactions, peristaltic movements, oxygen level control, among others, contribute to determining which microbes are able to grow and persist in the gut environment.

of administration and excretion, as well as on the residing gut microbiota. $^{862}$

Oral administration of antibiotics puts a prominent selective pressure on the gut microbiota due to direct effects on microbial growth, as well as alterations in the gut environment, such as reduction of the inner mucus layer, loss of antimicrobial peptides and immune tolerance, ${ }^{41}$ besides a decrease in the production of butyrate, a microbial metabolite important for the maintenance of anaerobiosis in the gut. ${ }^{47}$ Consequently, antibiotics perturbations can result in the replacement of the complex community of anaerobes, typical of the healthy adult gut microbiome, by a community of lower overall microbial diversity and increased abundance of facultative anaerobes, ${ }^{58}$ such as E. coli and Salmonella spp. ${ }^{59}$

Essentially, the degree and direction of alterations in response to antibiotic treatment are highly individual-specific. ${ }^{63} 64$ Supporting evidence shows that the initial composition of the gut microbiota, prior to antibiotic treatment, significantly determines the impact of the drug on the ecosystem. Lower initial microbiota diversity seems to be favourable to the enrichment of opportunistic pathogens as shown, for example, after cefprozil treatment. ${ }^{65}$ Contrary to that, the gut microbiota of healthy young adults seems to be resilient, as evidenced after an antibiotics intervention (meropenem, gentamicin and vancomycin administered orally for 4 days), indicating that the recovery of individual species in this case was modulated by their arsenal of antibiotic resistance genes. ${ }^{8}$

The overly exposed human microbiome has become a significant reservoir of resistance genes, ${ }^{66}$ which can persist in the microbial community long after the antibiotic therapy has ended, contributing to the increasing difficulty in controlling bacterial infections. Higher susceptibility to infections is one of the most imminent threats of gut microbiota alterations after antibiotic use, which can be a consequence of loss of colonisation resistance against pathogens. ${ }^{44}$ In particular, antibiotic-associated diarrhoea due to the nosocomial pathogen $C$. difficile occurs frequently. ${ }^{14}$ To overcome that, administration of microbial species to correct a clinically relevant microbiome deficiency, compensating for loss of original gut inhabitants after antibiotic treatment has been proposed. ${ }^{67}$ For example, colonisation resistance to C. difficile infection was restored in mice with the supplementation of C. scindens, via a secondary bile acid mediated process. ${ }^{68}$

Antibiotics with strong and broad activity against anaerobes, such as ciprofloxacin and clindamycin, typically cause longlasting effects on microbiota composition, for example with decrease in the relative abundance of Bifidobacterium in both treatments, as well as of Bacteroides in the clindamycin group. ${ }^{69}$ Interestingly, clindamycin-induced unhealthy state was minimised in a murine study with the administration of Lactobacillus paracasei CNCM I-3689, which promoted the recovery of Bacteroidetes members, and reduced the faecal level of vancomycinresistant enterococci. ${ }^{70}$

Depletion of butyrate-producing bacteria is considered one of the main drivers of the development of unhealthy states of the gut microbiome. Oral administration of vancomycin has been associated with reduction in the relative abundance of butyrate producers, such as Coprococcus eutactus and Faecalibacterium prausnitzii, accompanied by a systemically lower butyrate concentration in the plasma. ${ }^{63}$ Restoration of butyrate production in the gut could be achieved by either the administration of butyrate producers as probiotics, which are currently being 
developed, ${ }^{71}$ or with the supplementation of substrates to specifically promote their growth. ${ }^{72}$

Overall, bacterial diversity usually decreases during the week immediately following antibiotic therapy and then begins to recover in a secondary succession being capable of returning to a composition similar to the original one, but the initial state is often not fully recovered. ${ }^{5862}$ The response and recovery of the gut microbiota after antibiotics therapy seem to depend on four major aspects of the microbiome: (1) microbiota composition prior to treatment, (2) functional redundancy, (3) level of opportunistic pathogens and (4) level of antibiotic resistance genes. Therefore, here we review and devise strategies to counteract the impact of antibiotics, considering each of these factors (figure 4).

Despite the potential negative consequences of antibiotics therapy to human health, their use is the most reliable approach to treat bacterial infections. In this perspective, it would be particularly relevant to find ways to reduce their unwanted impacts on the gut microbiome, or to increase the resilience of the microbial ecosystem before antibiotic exposure. The development of a higher resilience state of the gut microbiome could be achieved by favouring the maintenance of the microbiota composition and stability during perturbations, or contributing to a faster recovery by either stimulating key microbial species involved in resilience mechanisms, or replacing microbes and functions that might have been lost in system. To this end, modulation with diet, including the supplementation with nondigestible carbohydrates (NDCs), ${ }^{73} 74$ the use of probiotics ${ }^{3770}$ or defined microbial communities, ${ }^{75}$ as well as faecal microbiota transplantation, ${ }^{11}{ }^{14}$ are promising avenues.

\section{Diet shaping gut microbiome resilience}

A balanced diet, according to the dietary recommendations, provides the right intake of macronutrients (carbohydrates, proteins and fat), as well as micronutrients (vitamins and minerals), resulting in homeostasis and human health. ${ }^{76}$ Longterm diet is strongly associated with the composition, activity and dynamics of the human gut microbiome, while short-term dietary changes are often not sufficient to elicit major changes in the ecosystem. ${ }^{3077}$ Since nutrients mediate fundamental processes in microbes and can be provided by a balanced diet, they represent a potential target for reshaping microbial communities.

Modulation of the human gut microbiome with dietary interventions has been extensively studied, mainly focusing on the supplementation of NDCs. ${ }^{777879}$ However, the impact of dietary components on the stability and resilience of the gut ecosystem has been barely addressed. This may be partially attributable to gaps that still exist in our knowledge about the underlying mechanisms.

From an ecological point of view, diet is one of the strongest selective pressures for microbial communities within the gut, ${ }^{54}$ which is partially mediated by competition for nutrients, and increased fitness by cross-feeding interactions. ${ }^{50}$ The ability to extract energy from nutrients that are non-digestible by the host provides an evolutionary driving force for microorganisms to maintain residency in the host gut. ${ }^{30}$ Notably, the highest concentration of microorganisms is found in the colon, which acts as a fermenter system for dietary compounds that escape digestion and absorption by the host. ${ }^{7}$ While the proximal colon has predominantly saccharolytic activity, protein fermentation increases distally, as well as $\mathrm{pH}$, through the transverse colon and into the distal colon. ${ }^{80}$

NDCs are quantitatively the main substrate for microbial growth in the colon. The capacity of degrading NDCs in the gut varies greatly among microbial species, depending on the presence of carbohydrate-active enzymes. ${ }^{81}$ Certain predominant Bacteroides species, for example, possess broad metabolic capacity, being able to switch readily between different NDCs available in the environment. ${ }^{43}$ On the other hand, nutritionally specialised bacteria appear to play critical roles in the community by initiating the degradation of complex substrates such as polymeric NDCs. ${ }^{51}$

Specific promotion of bacterial growth at the level of species and strains can be achieved through the administration of NDCs with prebiotic effects, thus altering the composition of the gut microbiota by selectively enriching for the desired microorganisms. This particular approach has been effectively applied in vivo, for example, with the supplementation of galactooligosaccharides after amoxicillin treatment, ${ }^{74}$ and human milk oligosaccharides such as $2^{\prime}$-fucosyllactose, exhibiting a bifidogenic effect in the gut of healthy adults. ${ }^{79}$ Furthermore, the supplementation of NDCs to promote SCFA production, particularly butyrate, for example aiming at the modulation of T2DM with non-resistant starch, ${ }^{78}$ or improving fat oxidation in overweight/obese men with the administration of inulin, ${ }^{72}$ has been successfully achieved. These results indicate the potential of NDC supplementation to modulate gut health after antibiotics therapy, since certain antibiotics have been shown to reduce the abundance of bifidobacteria and butyrate producers in the gut. ${ }^{63}$ In this context, in vitro tests with the addition of fructooligosaccharides and xylo-oligosaccharides, were able to minimise the effects of either amoxicillin, doxycycline or clindamycin on the gut microbiota, as both substrates modulated microbial composition, resulting in higher butyrate production. ${ }^{73}$

The increased consumption of a Western-style diet, low in NDCs, is believed to have selected for a microbiota with altered composition and functionality over time, including the loss of some carbohydrate-degrading species. ${ }^{82}$ When NDCs are limited, bacteria can turn to alternative energy sources from the diet, or may degrade host glycans present in the gut mucus layer, contributing to the development of inflammatory states associated with allergies, infections and autoimmune diseases. ${ }^{41}$ Other substrates that can be used by gut bacteria when NDCs are not available are proteins and fat. Fermentation of proteins results in the production of branched-chain fatty acids, ammonia, hydrogen sulfide, phenolic and indolic compounds, as well as amines and polyamines. ${ }^{83}$ These metabolites can be toxic for the gut and have been associated with obesity-related complications such as non-alcoholic fatty liver disease, insulin resistance and T2DM. ${ }^{12}$ Fat consumption, on the other hand, usually has indirect effects on the gut microbiome, mediated by the secondary metabolism of bile salts by bacteria in the colon, shaping gut microbiota composition due to their antimicrobial properties, acting against bacterial cell membranes and inducing DNA and protein damage.

Although less attention is given to vitamins and minerals, they seem to be equally important in the symbiotic relationship between host and microbes, and may also play a role in shaping microbial composition in the gut. Vitamin $\mathrm{K}$ and $\mathrm{B}$ group vitamins are available in the diet but can also be synthesised by gut bacteria and then shared among species via cross-feeding interactions. ${ }^{84}$ Competition for minerals, which are required cofactors for several human and microbial metabolic processes, can also determine the species able to grow and persist in the gut ecosystem. High iron levels in the gut, for example, have been associated with increased growth of pathogens. ${ }^{85}$

Altogether, a diet rich in NDCs, along with adequate intake of macro and micronutrients is associated with higher gut microbial 

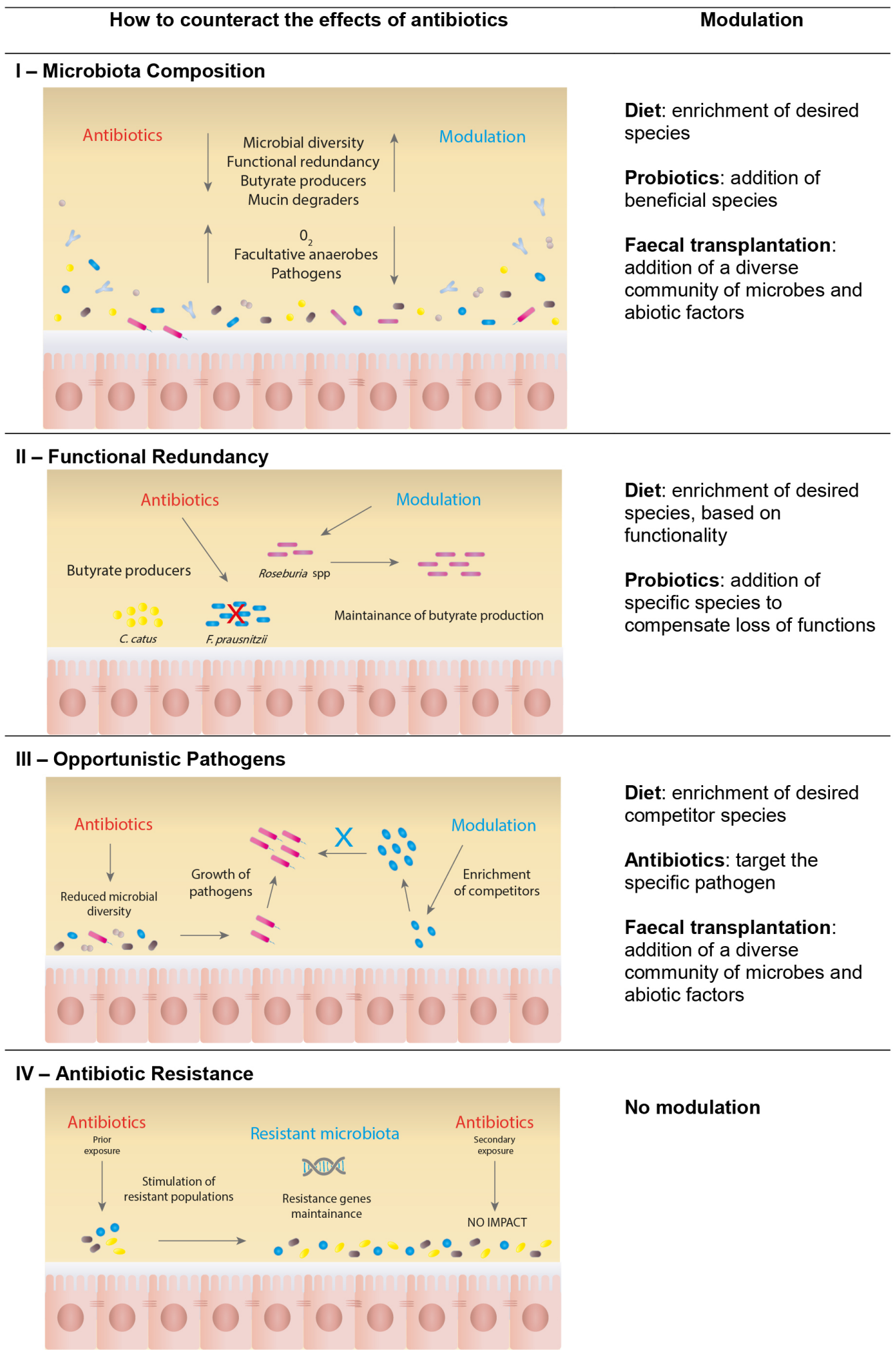

Figure 4 Approaches to counteract the effects of antibiotics on the human gut microbiome. Four main factors are known to play an essential role in the resilience of the human gut microbiome and its recovery after antibiotics perturbations. Specific approaches to counteract the impacts of antibiotics are listed on the right side. (I) Microbiota composition: high diversity seems to be associated with resilience of the gut ecosystem. Which species are present and their abundance also plays a crucial role. (II) Functional redundancy: different microbes have distinct functions in the gut ecosystem. Microbial diversity is essential for the maintenance of functions when one or more species are lost due to a perturbation. (III) Opportunistic pathogens: the number and abundance of potentially pathogenic species already present in the gut will determine their ability to expand after a perturbation. (IV) Antibiotic resistance: the level of antibiotic resistance genes in the gut can be critical for the response of the gut microbial community to new antibiotic perturbations. For a highly antibiotic-resistant microbiota, the impacts of a given antibiotic therapy would already be minimised in the ecosystem. However, modulatory strategies might be necessary in the case of expansion of antibiotic-resistant opportunistic pathogens. 
diversity and is considered beneficial for human health. ${ }^{80}$ On the contrary, a highly processed diet, rich in fat, refined sugars, salt and additives, and low in NDCs, is linked to a decreased microbial diversity in the gut, negatively affecting host health. ${ }^{3186}$ Reduced diversity usually is associated with the loss of key species essential to keep the equilibrium of the ecosystem, potentially impacting microbial resilience and resulting in disease. This has been evidenced by studies determining the gut microbiota composition of individuals when consuming a natural unprocessed diet, more similar to that of ancient populations, as compared with the modern industrialised diet. ${ }^{8687}$

The long-term benefits of consuming a natural, unprocessed diet appear to be the consensus among the majority of studies on human gut microbiome, nutrition and health. In addition to that the highly individual response to diet ${ }^{88} 89$ highlights the need for personalised recommendations, based on different gut microbiota compositions, as well as on distinct metabolic phenotypes. ${ }^{90}$

\section{Personalised dietary interventions for unique microbiomes}

To date, the disappointing efficacy of nutritional interventions to manage microbiota-associated diseases may stem from the lack of attention for interindividual variabilities in dietary responses and gut microbiota profile. ${ }^{1676}$ Another limitation is the supplementation of already processed NDCs, which seem to be less effective than the unprocessed alternatives and/or strategies combining slowly and rapidly fermentable NDCs.

The current need for translating microbiome research into targeted modulations has been discussed and acknowledged. A recent review ${ }^{17}$ highlighted the potential of applying precision microbiome diagnostics and management, described by the authors as the use of the gut microbiome as a biomarker to predict the effect of specific dietary components on host health and the use of these data to design precision diets and interventions that ensure optimal health at the level of individuals.

The future of microbiome-based precision nutrition shall comprise the expansion of next-generation 'omics' tools and sophisticated statistics methods, extensively applied for research purposes, to the clinical practice. ${ }^{91}$ This would include gut microbiome taxonomic and metagenomic profiling for identification of gaps in key microbes and their functions involved in the maintenance of stability in the healthy ecosystem, allowing the development of tailored strategies for supplementation or enrichment of these species and functions. Metaproteome analyses can be used to search for essential functions lost in the system, which can be restored by the administration of species known to produce the lacking proteins. Lastly, the absolute abundance of specific microbes associated with health and disease states can be assessed by approaches including quantitative PCR and fluorescent in situ hybridisation technologies. In parallel, for the assessment of host health, a detailed metabolic phenotypic evaluation may be performed, comprising measurements of body composition and tissue-specific insulin resistance, complementary to the classical global methods.

In the perspective of personalised microbiomes, the identification of high-level clusters, such as enterotypes, which aim at stratifying individuals based on their gut microbiota composition, can be considered a step forward in the direction of tailored approaches. ${ }^{4}$ In line with this, the concept of enterotypes paved the way for other efforts towards subject-specific therapies, exemplified by the two distinct strategies for weight loss proposed for either the Prevotella-enterotype or Bacteroidesenterotype, considering their different responses to NDCs. ${ }^{92}$
Similarly, recent studies proposed a treatment stratification based on host genome and gut microbiome for IBD, ${ }^{13}$ and the stratification of patients based on the composition of their gut microbiota for the prescription of cancer therapeutic drugs. ${ }^{93}$

The establishment of personalised recommendations and interventions will require a deeper knowledge of the factors shaping each individual's gut microbiota composition, to advance beyond the current strategies for stratification. Applying precision nutrition to improve the recovery of the gut ecosystem after perturbations will depend fundamentally on better understanding the mechanisms underlying stability and resilience. ${ }^{22}$ With this information, it will be possible to identify which dietary components, prebiotics or probiotics are likely to be the most effective for an individual patient, along with consideration of the optimal daily intakes.

\section{CONCLUSIONS}

Gut microbiota diversity and stability, as well as the set of microbial interactions and host-microbe symbioses, seem to be critical aspects for the resilience of healthy states. From a clinical, translational perspective, the ability to predict resilience of the human gut microbiome prior to perturbation, or to restore this property in degraded ecosystems, would offer significant benefits. One of the next steps is to further understand the microbial interactions and the functions of the microbiome, along with the mechanisms associated with health and disease. These results hold promise for novel treatment strategies, where individual gut microbiotas are characterised and therapies are personalised, either by targeting key bacteria or involving specific combinations of prebiotics and probiotics in order to restore ecosystem stability and function.

Unhealthy states of the gut microbiome can be resilient, possibly causing a predisposition to chronic diseases and a resistance to the efficacy of treatments. Although a balanced diet has been recognised as the main factor shaping gut microbiota composition, the current nutrition recommendations remain fundamentally general, not considering the particularity of host genetics and personalised microbiomes.

In order to formulate meaningful and clinically relevant dietary advice to improve gut microbiome resilience, studies on the microbiome, both in health and in disease, must integrate populationscale epidemiology with narrow but deep clinical studies in the setting of personalised nutrition, including approaches to help understanding the mechanisms behind individual responsiveness to modulatory interventions. Furthermore, future research should investigate beyond the single-nutrient approach, and focus on the effect of the whole diet on the gut microbiome.

Contributors MF searched literature, wrote the manuscript and created the figures. $E B, J P, A N, H S$ and $E G Z$ reviewed and wrote the manuscript. All authors approved the final manuscript.

Funding This work was performed in the partnership 'CarboBiotics', project number ALWCC.2017.003, coordinated and financed by the Dutch Research Council (NWO) and the Carbohydrate Competence Center (CCC), with the collaboration of the participating industrial partners: Avebe, Friesland Campina, Nutrition Sciences, and TKI Agri\&Food.

Competing interests None declared.

Patient consent for publication Not required.

Provenance and peer review Commissioned; externally peer reviewed.

ORCID iD

Erwin G Zoetendal http://orcid.org/0000-0002-7149-0727

\section{REFERENCES}

1 Gilbert JA, Blaser MJ, Caporaso JG, et al. Current understanding of the human microbiome. Nat Med 2018;24:392-400. 
2 Human Microbiome Project Consortium. Structure, function and diversity of the healthy human microbiome. Nature 2012;486:207-14.

3 Zoetendal EG, Akkermans ADL, Akkermans-van Vliet WM, et al. The host genotype affects the bacterial community in the human gastrointestinal tract. Microb Ecol Health Dis 2001;13:129-34.

4 Costea PI, Hildebrand F, Arumugam M, et al. Enterotypes in the landscape of gut microbial community composition. Nat Microbiol 2018;3:8-16.

5 Yatsunenko T, Rey FE, Manary MJ, et al. Human gut microbiome viewed across age and geography. Nature 2012;486:222-7.

6 David LA, Maurice CF, Carmody RN, et al. Diet rapidly and reproducibly alters the human gut microbiome. Nature 2014;505:559-63.

7 Rowland I, Gibson G, Heinken A, et al. Gut microbiota functions: metabolism of nutrients and other food components. Eur J Nutr 2018;57:1-24.

8 Palleja A, Mikkelsen KH, Forslund SK, et al. Recovery of gut microbiota of healthy adults following antibiotic exposure. Nat Microbiol 2018:3:1255-65.

9 Sommer F, Anderson JM, Bharti R, et al. The resilience of the intestinal microbiota influences health and disease. Nat Rev Microbiol 2017:15:630-8.

10 Duvallet C, Gibbons SM, Gurry T, et al. Meta-analysis of gut microbiome studies identifies disease-specific and shared responses. Nat Commun 2017:8:1784.

11 Kootte RS, Levin E, Salojärvi J, et al. Improvement of insulin sensitivity after lean donor feces in metabolic syndrome is driven by baseline intestinal microbiota composition. Cell Metab 2017;26:611-9.

12 Canfora EE, Meex RCR, Venema K, et al. Gut microbial metabolites in obesity, NAFLD and T2DM. Nat Rev Endocrinol 2019:15:261-73.

13 Moustafa A, Li W, Anderson EL, et al. Genetic risk, dysbiosis, and treatment stratification using host genome and gut microbiome in inflammatory bowel disease. Clin Trans/ Gastroenterol 2018;9:e132.

14 van Nood E, Vrieze A, Nieuwdorp M, et al. Duodenal infusion of donor feces for recurrent Clostridium difficile. N Eng/ J Med 2013;368:407-15.

15 Schmidt TSB, Raes J, Bork P. The human gut microbiome: from association to modulation. Cell 2018;172:1198-215.

16 Bashiardes S, Godneva A, Elinav E, et al. Towards utilization of the human genome and microbiome for personalized nutrition. Curr Opin Biotechnol 2018:51:57-63.

17 Mills S, Stanton C, Lane JA, et al. Precision nutrition and the microbiome, part I: current state of the science. Nutrients 2019;11:923.

18 Sender R, Fuchs S, Milo R. Are we really vastly outnumbered? Revisiting the ratio of bacterial to host cells in humans. Cell 2016:164:337-40.

19 Almeida A, Mitchell AL, Boland M, et al. A new genomic blueprint of the human gut microbiota. Nature 2019:568:499-504.

20 Qin J, Li R, Raes J, et al. A human gut microbial gene catalogue established by metagenomic sequencing. Nature 2010;464:59-65.

21 Martínez I, Muller CE, Walter J. Long-term temporal analysis of the human fecal microbiota revealed a stable core of dominant bacterial species. PLoS One 2013;8:e69621

22 Shetty SA, Hugenholtz F, Lahti L, et al. Intestinal microbiome landscaping: insight in community assemblage and implications for microbial modulation strategies. FEMS Microbiol Rev 2017;41:182-99.

23 Lozupone CA, Stombaugh JI, Gordon JI, et al. Diversity, stability and resilience of the human gut microbiota. Nature 2012;489:220-30.

24 Relman DA. The human microbiome: ecosystem resilience and health. Nutr Rev 2012;70:S2-9.

25 Faith JJ, Guruge JL, Charbonneau M, et al. The long-term stability of the human gut microbiota. Science 2013;341:1237439.

26 Shaw LP, Bassam H, Barnes $C P$, et al. Modelling microbiome recovery after antibiotics using a stability landscape framework. Isme J 2019;13:1845-56.

27 Lahti L, Salojärvi J, Salonen A, et al. Tipping elements in the human intestinal ecosystem. Nat Commun 2014:5:4344.

28 Dakos V, Matthews B, Hendry AP, et al. Ecosystem tipping points in an evolving world. Nat Ecol Evol 2019:3:355-62.

29 Scheffer M, Bascompte J, Brock WA, et al. Early-warning signals for critical transitions. Nature 2009;461:53-9.

30 Flint HJ, Duncan SH, Louis P. The impact of nutrition on intestinal bacterial communities. Curr Opin Microbiol 2017:38:59-65.

31 Le Chatelier E, Nielsen T, Qin J, et al. Richness of human gut microbiome correlates with metabolic markers. Nature 2013;500:541-6.

32 Blakeley-Ruiz JA, Erickson AR, Cantarel BL, et al. Metaproteomics reveals persistent and phylum-redundant metabolic functional stability in adult human gut microbiomes of Crohn's remission patients despite temporal variations in microbial taxa, genomes, and proteomes. Microbiome 2019;7:18

33 Foster KR, Schluter J, Coyte KZ, et al. The evolution of the host microbiome as an ecosystem on a leash. Nature 2017;548:43-51.

34 Coyte KZ, Schluter J, Foster KR. The ecology of the microbiome: networks, competition, and stability. Science 2015;350:663-6.

35 De Sordi L, Lourenço M, Debarbieux L. The battle within: interactions of bacteriophages and bacteria in the gastrointestinal tract. Cell Host Microbe 2019;25:210-8

36 Burgess SL, Gilchrist CA, Lynn TC, et al. Parasitic protozoa and interactions with the host intestinal microbiota. Infect Immun 2017;85:e00101-17.
37 Buffie CG, Pamer EG. Microbiota-mediated colonization resistance against intestinal pathogens. Nat Rev Immunol 2013;13:790-801.

38 Tytgat HLP, Nobrega FL, van der Oost J, et al. Bowel biofilms: tipping points between a healthy and compromised gut? Trends Microbiol 2019:27:17-25.

39 Swidsinski A, Weber J, Loening-Baucke V, et al. Spatial organization and composition of the mucosal flora in patients with inflammatory bowel disease. J Clin Microbiol 2005;43:3380-9.

40 Schluter J, Nadell CD, Bassler BL, et al. Adhesion as a weapon in microbial competition. Isme J 2015;9:139-49.

41 Wells JM, Brummer RJ, Derrien M, et al. Homeostasis of the gut barrier and potential biomarkers. Am J Physiol Gastrointest Liver Physiol 2017;312:G171-93.

42 Belzer C, Chia LW, Aalvink S, et al. Microbial metabolic networks at the mucus layer lead to diet-independent butyrate and vitamin $B_{12}$ production by intestinal symbionts. mBio 2017;8:e00770-00717.

43 Porter NT, Luis AS, Martens EC. Bacteroides thetaiotaomicron. Trends Microbiol 2018;26:966-7.

44 Kim S, Covington A, Pamer EG. The intestinal microbiota: antibiotics, colonization resistance, and enteric pathogens. Immunol Rev 2017;279:90-105

45 Sassone-Corsi M, Nuccio S-P, Liu H, et al. Microcins mediate competition among Enterobacteriaceae in the inflamed gut. Nature 2016;540:280-3.

46 Koh A, De Vadder F, Kovatcheva-Datchary P, et al. From dietary fiber to host physiology: short-chain fatty acids as key bacterial metabolites. Cell 2016:165:1332-45.

47 Rivera-Chávez F, Zhang LF, Faber F, et al. Depletion of butyrate-producing clostridia from the gut microbiota drives an aerobic luminal expansion of Salmonella. Cell Host Microbe 2016;19:443-54

48 Papenfort K, Bassler BL. Quorum sensing signal-response systems in gram-negative bacteria. Nat Rev Microbiol 2016;14:576-88.

49 Thompson JA, Oliveira RA, Djukovic A, et al. Manipulation of the quorum sensing signal Al-2 affects the antibiotic-treated gut microbiota. Cell Rep 2015;10:1861-71.

50 Morris BEL, Henneberger $\mathrm{R}$, Huber $\mathrm{H}$, et al. Microbial syntrophy: interaction for the common good. FEMS Microbiol Rev 2013;37:384-406.

51 Milani C, Lugli GA, Duranti S, et al. Bifidobacteria exhibit social behavior through carbohydrate resource sharing in the gut. Sci Rep 2015;5:15782.

52 Wolf PG, Biswas A, Morales SE, et al. H2 metabolism is widespread and diverse among human colonic microbes. Gut Microbes 2016;7:235-45.

53 Atashgahi S, Shetty SA, Smidt H, et al. Flux, impact, and fate of halogenated xenobiotic compounds in the gut. Front Physiol 2018;9:888.

54 Byndloss MX, Pernitzsch SR, Bäumler AJ. Healthy hosts rule within: ecological forces shaping the gut microbiota. Mucosal Immunol 2018;11:1299-305.

55 Beasley DE, Koltz AM, Lambert JE, et al. The evolution of stomach acidity and its relevance to the human microbiome. PLoS One 2015;10:e0134116.

56 Lloyd-Price J, Abu-Ali G, Huttenhower C. The healthy human microbiome. Genome Med 2016;8:51.

57 Tiffany CR, Bäumler AJ. Dysbiosis: from fiction to function. Am J Physiol Gastrointest Liver Physiol 2019:317:G602-8.

58 Kriss M, Hazleton KZ, Nusbacher NM, et al. Low diversity gut microbiota dysbiosis: drivers, functional implications and recovery. Curr Opin Microbiol 2018:44:34-40.

59 Litvak Y, Byndloss MX, Tsolis RM, et al. Dysbiotic Proteobacteria expansion: a microbial signature of epithelial dysfunction. Curr Opin Microbiol 2017;39:1-6.

60 Brüssow H. Problems with the concept of gut microbiota dysbiosis. Microb Biotechnol 2020;13:423-34.

61 Bhalodi AA, van Engelen TSR, Virk HS, et al. Impact of antimicrobial therapy on the gut microbiome. J Antimicrob Chemother 2019;74:i6-15.

62 Pérez-Cobas AE, Gosalbes MJ, Friedrichs A, et al. Gut microbiota disturbance during antibiotic therapy: a multi-omic approach. Gut 2013;62:1591-601.

63 Reijnders D, Goossens GH, Hermes GDA, et al. Effects of gut microbiota manipulation by antibiotics on host metabolism in obese humans: a randomized double-blind placebo-controlled trial. Cell Metab 2016:24:63-74.

64 Hildebrand F, Moitinho-Silva L, Blasche S, et al. Antibiotics-induced monodominance of a novel gut bacterial order. Gut 2019:68:1781-90.

65 Raymond F, Ouameur AA, Déraspe $M$, et al. The initial state of the human gut microbiome determines its reshaping by antibiotics. Isme J 2016;10:707-20.

66 Willmann M, Vehreschild MJGT, Biehl LM, et al. Distinct impact of antibiotics on the gut microbiome and resistome: a longitudinal multicenter cohort study. BMC Biol 2019:17:76.

67 Pamer EG. Resurrecting the intestinal microbiota to combat antibiotic-resistant pathogens. Science 2016;352:535-8.

68 Buffie CG, Bucci V, Stein RR, et al. Precision microbiome reconstitution restores bile acid mediated resistance to Clostridium difficile. Nature 2015;517:205-8.

69 Rashid M-U, Weintraub A, Nord CE. Development of antimicrobial resistance in the normal anaerobic microbiota during one year after administration of clindamycin or ciprofloxacin. Anaerobe 2015;31:72-7.

70 Crouzet L, Derrien M, Cherbuy C, et al. Lactobacillus paracasei CNCM 1-3689 reduces vancomycin-resistant Enterococcus persistence and promotes Bacteroidetes resilience in the gut following antibiotic challenge. Sci Rep 2018;8:5098. 
71 Martín R, Miquel S, Benevides L, et al. Functional characterization of novel Faecalibacterium prausnitzii strains isolated from healthy volunteers: a step forward in the use of $F$. prausnitzii as a next-generation probiotic. Front Microbiol 2017;8:1226.

72 van der Beek CM, Canfora EE, Kip AM, et al. The prebiotic inulin improves substrate metabolism and promotes short-chain fatty acid production in overweight to obese men. Metabolism 2018;87:25-35.

73 Míguez B, Gómez B, Parajó JC, et al. Potential of fructooligosaccharides and xylooligosaccharides as substrates to counteract the undesirable effects of several antibiotics on elder fecal microbiota: a first in vitro approach. J Agric Food Chem 2018;66:9426-37.

74 Ladirat SE, Schoterman MHC, Rahaoui H, et al. Exploring the effects of galactooligosaccharides on the gut microbiota of healthy adults receiving amoxicillin treatment. Br J Nutr 2014;112:536-46.

75 Elzinga J, van der Oost J, de Vos WM, et al. The use of defined microbial communities to model host-microbe interactions in the human gut. Microbiol Mol Biol Rev 2019;83:e00054-18.

76 Bechthold A, Boeing H, Tetens I, et al. Perspective: food-based dietary guidelines in Europe - scientific concepts, current status, and perspectives. Adv Nutr 2018:9:544-60

77 Wu GD, Chen J, Hoffmann C, et al. Linking long-term dietary patterns with gut microbial enterotypes. Science 2011;334:105-8.

78 Zhao L, Zhang F, Ding X, et al. Gut bacteria selectively promoted by dietary fibers alleviate type 2 diabetes. Science 2018;359:1151-6.

79 Elison E, Vigsnaes LK, Rindom Krogsgaard L, et al. Oral supplementation of healthy adults with 2'-O-fucosyllactose and lacto-N-neotetraose is well tolerated and shifts the intestinal microbiota. Br J Nutr 2016;116:1356-68.

80 Zoetendal EG, de Vos WM. Effect of diet on the intestinal microbiota and its activity. Curr Opin Gastroenterol 2014;30:189-95.

81 Oliphant K, Allen-Vercoe E. Macronutrient metabolism by the human gut microbiome: major fermentation by-products and their impact on host health. Microbiome 2019;7:91.

82 Sonnenburg ED, Sonnenburg JL. Starving our microbial self: the deleterious consequences of a diet deficient in microbiota-accessible carbohydrates. Cell Metab 2014;20:779-86.
83 Russell WR, Duncan SH, Scobbie L, et al. Major phenylpropanoid-derived metabolites in the human gut can arise from microbial fermentation of protein. Mol Nutr Food Res 2013;57:523-35.

84 LeBlanc JG, Milani C, de Giori GS, et al. Bacteria as vitamin suppliers to their host: a gut microbiota perspective. Curr Opin Biotechnol 2013;24:160-8.

85 Jaeggi T, Kortman GAM, Moretti D, et al. Iron fortification adversely affects the gut microbiome, increases pathogen abundance and induces intestinal inflammation in Kenyan infants. Gut 2015;64:731-42.

86 O'Keefe SJD, Li JV, Lahti L, et al. Fat, fibre and cancer risk in African Americans and rural Africans. Nat Commun 2015;6:6342.

87 Fragiadakis GK, Smits SA, Sonnenburg ED, et al. Links between environment, diet, and the hunter-gatherer microbiome. Gut Microbes 2019;10:216-27.

88 Johnson AJ, Vangay P, Al-Ghalith GA, et al. Daily sampling reveals personalized dietmicrobiome associations in humans. Cell Host Microbe 2019;25:789-802.

89 Healey GR, Murphy R, Brough L, et al. Interindividual variability in gut microbiota and host response to dietary interventions. Nutr Rev 2017;75:1059-80.

90 Trouwborst I, Bowser SM, Goossens GH, et al. Ectopic fat accumulation in distinct insulin resistant phenotypes; targets for personalized nutritional interventions. Front Nutr 2018;5:77.

91 Petrosino JF. The microbiome in precision medicine: the way forward. Genome Med 2018;10:12.

92 Christensen L, Roager HM, Astrup A, et al. Microbial enterotypes in personalized nutrition and obesity management. Am J Clin Nutr 2018;108:645-51.

93 Jobin C. Precision medicine using microbiota. Science 2018:359:32-4.

94 Marchesi JR, Ravel J. The vocabulary of microbiome research: a proposal. Microbiome 2015:3:31

95 Gibson GR, Hutkins R, Sanders ME, et al. Expert consensus document: the International Scientific Association for Probiotics and Prebiotics (ISAPP) consensus statement on the definition and scope of prebiotics. Nat Rev Gastroenterol Hepatol 2017;14:491-502.

96 Hill C, Guarner F, Reid G, et al. Expert consensus document. The International Scientific Association for Probiotics and Prebiotics consensus statement on the scope and appropriate use of the term probiotic. Nat Rev Gastroenterol Hepatol 2014;11:506-14. 